\title{
Expression of stem cell marker and receptor kinase genes in glioblastoma tissue quantified by real-time RT-PCR
}

\author{
Koji Yoshimoto $\cdot$ Xinlong Ma $\cdot$ Yaulei Guan $\cdot$ Masahiro Mizoguchi $\cdot$ \\ Akira Nakamizo • Toshiyuki Amano • Nobuhiro Hata • Daisuke Kuga • \\ Tomio Sasaki
}

Received: 14 September 2010/Accepted: 26 May 2011/Published online: 21 June 2011

(C) The Author(s) 2011. This article is published with open access at Springerlink.com

\begin{abstract}
Glioblastoma is dependent on a specific signaling pathway to maintain its tumor phenotype. The receptor tyrosine kinase (RTK) family mediates the multiple oncogenic growth factor receptor signaling and contributes to the pathogenesis of glioblastoma. Recently, many studies have shown that the expression of stem cell marker in glioblastoma tissue has prognostic significance, which indicates that the quantification of stem cell markers and RTK genes yields biological information about glioblastoma. In this study, we quantified RNA expression levels of stem cell markers [CD133, Nestin, BMI-1, maternal embryonic leucine zipper kinase (MELK), and Notch1-4] as well as RTKs (EGFR, ErbB4, VEGFR1-3, FGFR1, -2, PDGFRA, and PDGFRB) in 42 clinical samples of glioblastoma by the real-time RT-PCR method. We demonstrated that the expression of MELK is exclusively upregulated in glioblastoma tissue. Notch receptor expression is moderately upregulated and is correlated with that of VEGFR2, VEGFR3, and PDGFR $\beta$. Unsupervised clustering identified one unique sample group that showed high expression of most of the genes analyzed. Our results suggest that quantification of these stem cell markers and RTK genes can stratify patients based on the expression profile, which might provide insight into the glioma biology in each cluster.
\end{abstract}

K. Yoshimoto $(\bowtie) \cdot$ X. Ma · Y. Guan · M. Mizoguchi · A. Nakamizo - T. Amano - N. Hata - D. Kuga - T. Sasaki Department of Neurosurgery, Graduate School of Medical Sciences, Kyushu University, 3-1-1 Maidashi, Higashi-Ku, Fukuoka 812-8582, Japan

e-mail: kyoshimo@ns.med.kyushu-u.ac.jp
Keywords Glioma $\cdot$ Stem cell marker $\cdot$ Quantification

\section{Introduction}

Glioblastoma is the most common malignant primary brain tumor in adults. Despite surgery, radiation, and chemotherapy, the median survival after diagnosis is approximately 12 months. As shown by the Cancer Genome Atlas pilot project, receptor tyrosine kinase (RTK) signaling pathways such as epidermal growth factor receptor (EGFR) and platelet-derived growth factor receptor (PDGFR) are frequently altered and activated in glioblastomas [1]. Drugs targeting these oncogenic signaling pathways have shown clinical activity in patients with glioblastomas, and targeted therapy represents a promising new paradigm for the treatment of glioblastomas [2,3]. Thus, it is of particular importance to evaluate the activation of each RTK pathway in glioblastomas for the development of effective molecular targeted therapy.

It has been recently reported that the expression of some stem cell markers in glioblastoma has prognostic significance [4-9]. Although Nestin and CD133, which are the most accredited, are useful stem cell markers, they are known to be expressed also in non-stem cell populations and play an important role in gliomagenesis. Notch, maternal embryonic leucine zipper kinase (MELK), and BMI-1 (a polycomb gene) are other signaling molecules implicated in the stem cell signaling pathway [10-12]. However, the expression profiles and functional roles of these molecules in glioblastoma tissue have not been systematically investigated. We hypothesized that exploring the stem cell signaling pathway in conjunction with the RTK pathway in glioblastoma tissues would yield prognostic information for patients in the clinic. Furthermore, it 
may be possible that the stem cell signaling pathway as well as the RTK pathway could be molecular targets for a new treatment strategy.

Recent advancements in microarray technology have enabled us to investigate genome-wide molecular profiling and identify a new molecular target. This high-throughput technology, however, is expensive, and it cannot always be integrated into the clinical setting, where methodology that is easier to use and more cost effective is preferred. Real-time reverse transcription-polymerase chain reaction (RT-PCR) technology is easily integrated in the clinic. In this study, we quantified the expression of stem cell marker genes and RTK genes in glioma tissue using a real-time RT-PCR method, performed unsupervised hierarchal clustering, and evaluated the statistical correlation of these expression data.

\section{Materials and methods}

Tumor specimens

We collected 42 case samples of glioblastoma from the Kyushu University Brain Tumor Bank; these samples were obtained from patients during surgery under the approval of the ethics committee of our university. For all tumors, a histological diagnosis of glioblastoma was determined based on World Health Organization criteria by boardcertified neuropathologists. All 42 cases were primary glioblastomas; secondary glioblastomas and recurrent cases were excluded from this study. Normal brain reference RNA (NBRR) was purchased from Ambion and used as an internal control.

Target genes and primer design

Concerning stem cell marker genes, we evaluated the expression of CD133 and Nestin, which are currently the most accredited markers for the identification of neural stem cells. In addition, MELK and BMI-1 were included in this study because these genes contribute to stem cell maintenance. Notch receptors (Notch1-4) were also included in this study because the Notch signaling pathway is implicated in the maintenance of neural progenitors and in the generation of glia during development of the brain and is altered in gliomas [13, 14]. Of all the RTK genes, we selected nine genes that have been shown to be involved in tumor growth and proliferation in glioblastomas: EGFR, ErbB4, VEGFR1, VEGFR2, VEGFR3, FGFR1, EGFR2, PDGFRA, and PDGFRB. In total, 17 genes were included in this study. Amplification primer pairs were designed for nonredundant regions on the NCBI Reference Sequence (RefSeq) using Primer 3 software. The target genes and corresponding RefSeq are listed in Table 1.
Table 1 List of genes analyzed in this study

\begin{tabular}{lll}
\hline Gene & Chromosome map & $\begin{array}{l}\text { Reference sequence } \\
\text { (Ref Seq) }\end{array}$ \\
\hline EGFR & $7 \mathrm{p} 12$ & NM_005228 \\
ERBB4 & $2 \mathrm{q} 33$ & NM_001042599 \\
PDGFRA & $4 \mathrm{q} 12$ & NM_006206 \\
PDGFRB & $5 \mathrm{q} 31$ & NM_002609 \\
FGFR1 & $8 \mathrm{p} 12$ & NM_015850 \\
FGFR2 & $10 \mathrm{q} 26$ & NM_000141 \\
VEGFR1 & $13 \mathrm{q} 12$ & NM_002019 \\
VEGFR2 & $4 \mathrm{q} 11$ & NM_002253 \\
VEGFR3 & $5 \mathrm{q} 35$ & NM_002020.4 \\
Notch1 & $9 \mathrm{q} 34$ & NM_017617 \\
Notch2 & $1 \mathrm{p} 12$ & NM_024408 \\
Notch3 & $19 \mathrm{p} 13$ & NM_000435 \\
Notch4 & $6 \mathrm{p} 21$ & NM_004557 \\
Nestin & $1 \mathrm{q} 23$ & NM_006617.1 \\
MELK & $9 \mathrm{p} 13$ & NM_014791 \\
BMI-1 & $10 \mathrm{p} 13$ & NM_005180 \\
CD133 & $4 \mathrm{p} 15$ & NM_006017 \\
\hline
\end{tabular}

RNA extraction, reverse transcription, and real-time reverse transcription-polymerase chain reaction

Total RNA was extracted from the frozen samples using a commercial mirVana miRNA isolation kit (Ambion, Austin, TX, USA). Reverse transcriptions were performed by random hexamers using a High Capacity cDNA Reverse Transcription kit (Applied Biosystems, Carlsbad, CA, USA). Reverse-transcribed products were amplified by the SYBR method using the ABI PRISM 7500 Fast Real-time PCR System (Applied Biosystems). Briefly, $2 \mu \mathrm{l}$ cDNA product was used as a template in a $20-\mu \mathrm{l}$ PCR reaction containing $10 \mu$ l Power SYBR Green Master Mix (Applied Biosystems), $200 \mathrm{mM}$ each primer, $1 \mu \mathrm{l}$ dimethylsulfoxide (DMSO), and $6.2 \mu \mathrm{l}$ distilled water. Hypoxanthine phosphoribosyltransferase 1 (HPRT1) was used as a reference gene. Amplification protocols were as follows: $95^{\circ} \mathrm{C}$ for $10 \mathrm{~min}, 40$ cycles of $95^{\circ} \mathrm{C} / 15 \mathrm{~s}$, and 40 cycles of $60^{\circ} \mathrm{C} / 60 \mathrm{~s}$ with melting curve analysis. Threshold cycle number (CT) was automatically determined by ABI 7500 Fast System SDS software. All reactions were performed in duplicate.

Data analysis of quantitative RT-PCR and statistical analysis

The average CT value of duplicate runs was determined for each gene, and $\Delta \mathrm{CT}$ was calculated by subtracting the $\mathrm{CT}$ value of the HPRT1 reference gene from that of each gene. The relative expression of each gene was quantified by the $\Delta \Delta \mathrm{CT}$ method. In this method, $\Delta \Delta \mathrm{CT}$ was calculated by 
Fig. 1 The relative quantity value $(R Q)$ normalized by normal brain reference RNA is shown as a log-transformed number for 17 genes. The bottom and top of the box indicate lower and upper quartiles, respectively. The band at the middle of the box indicates the median value of all samples. Upper and lower bars indicate the upper 90th percentile and lower 10th percentile, respectively. For each gene, outliers of this range were not plotted in this figure

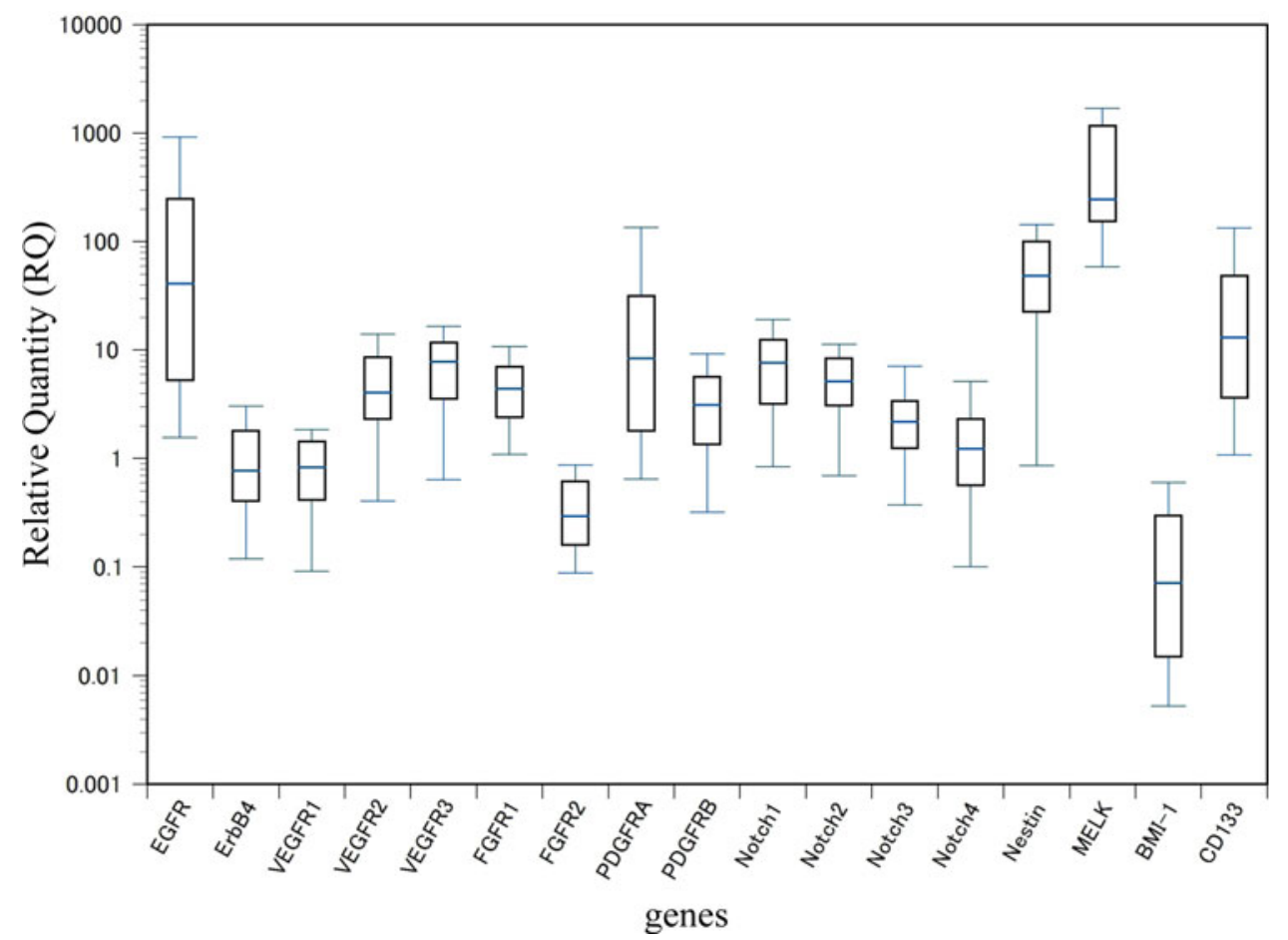

subtracting the $\Delta \mathrm{CT}$ of the NBRR from that of the target gene, and the relative quantity (RQ) was calculated. To auto-scale the data for clustering, the RQ of each gene was divided by the mean RQ value of all samples, and the data were presented as $\log _{10}$ of this value. Hierarchical clustering using Ward's linkage analysis and Spearman's rank test was performed by JMP 8 statistical software (SAS Institute, Cary, NC, USA).

\section{Results}

Quantification of stem cell markers and RTK genes

Amplified products were successfully obtained for all genes. The CT value of NBRR was less than 35 in all genes except MELK, for which the CT value was 38.4, indicating that MELK expression is very low or absent in healthy brain tissue. The RQ value was box-plotted as log-transformed numbers (Fig. 1). For each gene, outlying cases in the upper and lower $10 \%$ of expression were not plotted in this figure. In glioblastoma tissues, EGFR, PDGFRA, Nestin, MELK, and CD133 are strongly expressed compared to normal brain tissue. Notably, MELK expression is exclusively upregulated in glioblastoma tissue without quantifiable expression in normal brain tissue. The expression of VEGFR2, VEGFR3, FGFR1, PDGFRB, and Notch1-3 are slightly higher than that in normal brain tissue. Notably, the stem cell marker BMI-1 showed decreased expression in glioblastoma tissues. In total, the median RQ value of ErbB4, VEGFR1, FGFR2, and BMI-1 was less than 1 , which showed that the expression level of each of these genes is less than or equal to that in normal brain tissue.

Hierarchical clustering categorized glioma tissues into three clusters

To compare the expression profiles of the different samples and detect groups of samples with similar expression profiles, we performed hierarchical clustering of the expression data of the 13 genes (excluding ErbB4, VEGFR1, FGFR2, and BMI-1) with higher expression compared with normal brain tissue. The dendrogram of this clustering demonstrated that all 42 glioma tissues could be categorized into three clusters (Fig. 2). Although we could not conclude the expression profile of samples within cluster 1 , cluster 2 showed a high level of expression of almost all genes including stem cell markers and RTKs, and cluster 3 showed a low level of expression of all genes.

Spearman's rank test of genes with strong correlation of expression

To evaluate the correlation of the expression patterns among these 13 genes, we performed pairwise Spearman's correlation tests. Strong correlation was detected among Notch1-4, PDGFRB, VEGFR2, and VEGFR3 (Table 2. There were no significant correlations among stem cell markers. 


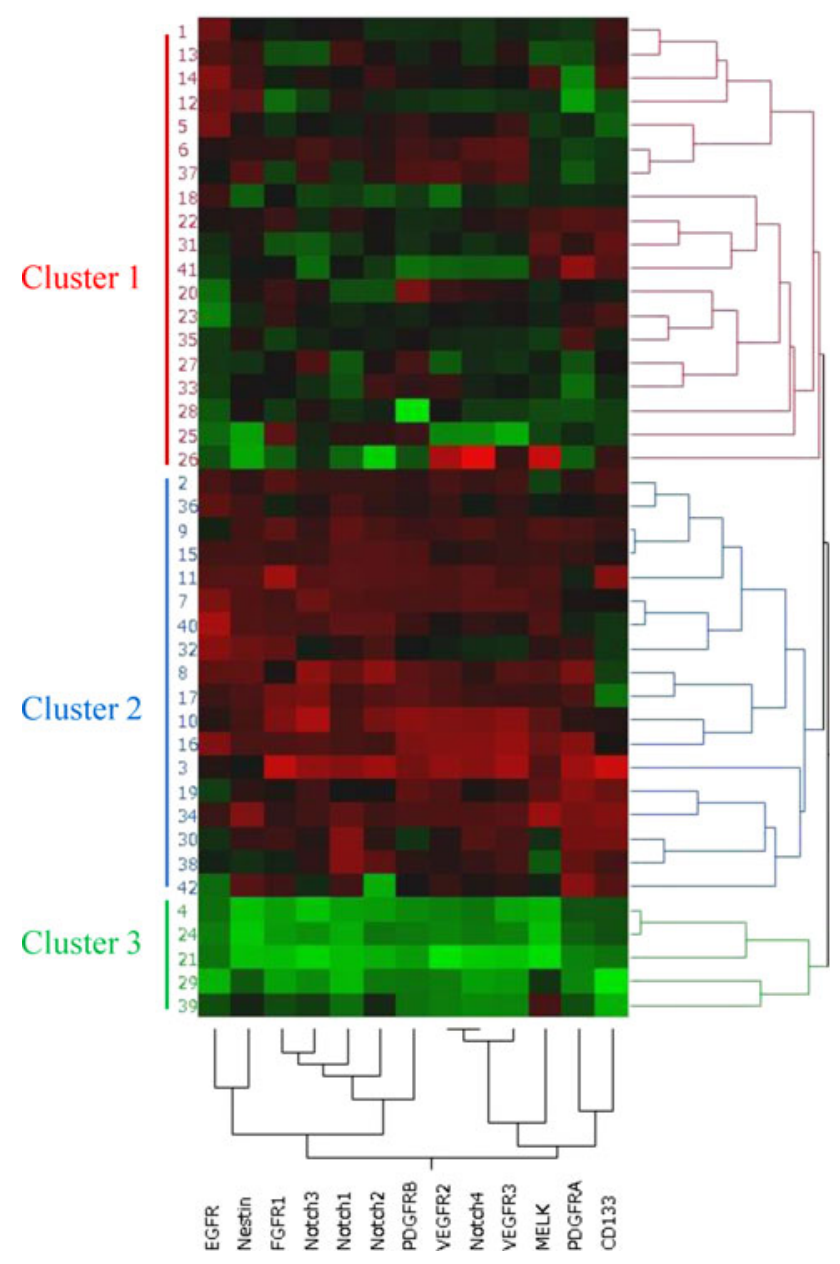

Fig. 2 Hierarchical clustering analysis demonstrated that all the samples could be classified into three clusters based on the analysis of 13 genes. In cluster 2, samples show high expression of most of the genes. By contrast, expression of all the genes is low in cluster 3

\section{Discussion}

Our results demonstrated that the expression of MELK, an atypical member of the snfl/AMPK family of serine-threonine kinases, which are key regulators of the proliferation and maintenance of glioma stem cells, is exclusively upregulated in glioblastoma tissues in contrast to Nestin, CD133, and Notch, expression of which is also detected in normal brain tissue. The RQ of MELK expression is the same or higher than that of EGFR, which has been known to be overexpressed in glioblastoma tissue. It has been also reported that MELK expression is positively increased according to tumor grade [15]. Our results therefore imply that the MELK signaling pathway can be a therapeutic target for glioblastomas.

The relative quantification of RTK genes in this study is consistent with previous studies [16-19]. EGFR is the most highly expressed gene, followed by PDGFRA. The expression of PDGFRB is lower than that of PDGFRA, and
Table 2 List of gene combinations with strong correlation of gene expression

\begin{tabular}{ll}
\hline Genes & $\begin{array}{l}\text { Correlation } \\
\text { coefficient }\end{array}$ \\
\hline Notch4 versus VEGFR3 & 0.92 \\
Notch4 versus VEGFR2 & 0.88 \\
VEGFR3 versus VEGFR2 & 0.88 \\
Notch3 versus PDGFRB & 0.84 \\
Notch2 versus Notch1 & 0.79 \\
Notch3 versus Notch2 & 0.79 \\
Notch3 versus VEGFR3 & 0.78 \\
Notch3 versus Notch4 & 0.78 \\
Notch3 versus VEGFR2 & 0.74 \\
Notch1 versus VEGFR3 & 0.73 \\
PDGFRB versus VEGFR2 & 0.73 \\
Notch2 versus PDGFRB & 0.72 \\
PDGFRB versus VEGFR3 & 0.72 \\
\hline
\end{tabular}

Correlation coefficient was obtained by Spearman's rank test. All these correlation coefficients were statistically significant $(P<0.0001)$

VEGFR2 and -3 are more highly expressed than VEGFR1. Regarding other stem cell markers, Nestin and CD133 [20], the two most accredited stem cell markers, are expressed in the same range, although their expression is not tumor specific, which suggests that the biological significance of the expression of these markers should be thoroughly investigated. The Notch pathway is a conserved ligandreceptor signaling mechanism that modulates cell fate and differentiation and plays an important role in the maintenance of stem cell self-renewal. In mammals, there are four Notch receptors (Notch1-4) and five ligands (Jagged1, -2; Delta-like1, -3, -4) [14]. Although the key components of this Notch signaling are reported to be aberrantly activated in gliomas and are considered to be implicated in gliomagenesis, the quantification of these four Notch receptors in gliomas has been inadequately investigated [21]. Our results demonstrated that Notch 1 and Notch 2 are upregulated by 5- to 20 fold compared with normal tissue, whereas the expression of Notch3 and Notch4 is threefold higher on average, which might suggest that Notch1 and Notch2 signaling are more important than that of Notch3 and Notch4. Conversely, the results of BMI-1 expression in our study showed a discrepancy with previous studies. Although it has been reported that BMI-1 inhibits activation of the tumor suppressor pathway and its expression is highly upregulated in glioblastomas [22], our results revealed that BMI-1 expression is not upregulated compared with normal tissue. Further study is needed to address this discrepancy.

Our clustering data demonstrated that 42 glioma tissues could be classified into three groups based on the 
expression of 13 stem cell markers and RTK genes. The demonstrative feature of this clustering is that gliomas in cluster 2 show relatively high expression of all genes, whereas the expression of all genes is relatively low in cluster 3. Further studies are needed to evaluate whether this subclassification can be applied to larger sample sets and whether this clustering has prognostic significance, because we have not included prognostic information in this study. Furthermore, because a recent study has reported a glioblastoma molecular stratification defined as proneural, neural, classical, and mesenchymal based on genomic alteration [23-25], we need to evaluate how each unique cluster falls into these categories.

The clustering data also give us a general description of how the selected genes co-varied with respect to their expression level, and some of the correlation is confirmed by statistical analysis. Pairwise Spearman's correlation analysis on all genes revealed high correlation coefficients in some pairs (see Table 2). Notably, genes listed in Table 2 are all implicated in the angiogenesis of normal and tumor tissue. We did not see strong statistical correlations among stem cell markers MELK, Nestin, CD133, and Notch receptors. In addition, there is a possibility that gene pairs with moderate correlation may have been missed with these criteria, because we have only listed the gene pairs for which the correlation coefficient is more than 0.7. In fact, the correlation coefficient of EGFR and Nestin, and of PDGFRA and CD133, both of which are in the same gene tree in the dendrogram, was $0.68(P<0.0001)$ and $0.55(P=0.0002)$, respectively.

In summary, we demonstrated that the expression of MELK is exclusively upregulated in glioblastoma tissue. Although Notch receptor expression is moderately upregulated in glioblastoma tissue, its expression showed a strong correlation with angiogenesis-related genes VEGFR2, VEGFR3, and PDGFRB. We need to address the biological and prognostic features of glioblastomas grouped within cluster 2 because this group showed the unique findings that all the genes are highly expressed.

Open Access This article is distributed under the terms of the Creative Commons Attribution Noncommercial License which permits any noncommercial use, distribution, and reproduction in any medium, provided the original author(s) and source are credited.

\section{References}

1. Cancer Genome Atlas Research Network (2008) Comprehensive genomic characterization defines human glioblastoma genes and core pathways. Nature (Lond) 455:1061-1068

2. Rich JN, Bigner DD (2004) Development of novel targeted therapies in the treatment of malignant glioma. Nat Rev Drug Discov 3:430-446
3. Mellinghoff IK, Wang MY, Vivanco I et al (2005) Molecular determinants of the response of glioblastomas to EGFR kinase inhibitors. N Engl J Med 353:2012-2024

4. Nicolis SK (2007) Cancer stem cells and "stemness" genes in neuro-oncology. Neurobiol Dis 25:217-229

5. Zeppernick F, Ahmadi R, Campos B et al (2008) Stem cell marker CD133 affects clinical outcome in glioma patients. Clin Cancer Res 14:123-129

6. Ma YH, Mentlein R, Knerlich F et al (2008) Expression of stem cell markers in human astrocytomas of different WHO grades. J Neuro-oncol 86:31-45

7. Dell'Albani P (2008) Stem cell markers in gliomas. Neurochem Res 33:2407-2415

8. Natsume A, Kinjo S, Yuki K et al (2011) Glioma-initiating cells and molecular pathology: implications for therapy. Brain Tumor Pathol 28:1-12

9. Strojnik T, Rosland GV, Sakariassen PO et al (2007) Neural stem cell markers, nestin and musashi proteins, in the progression of human glioma: correlation of nestin with prognosis of patient survival. Surg Neurol 68:133-143

10. Nakano I, Paucar AA, Bajpai R et al (2005) Maternal embryonic leucine zipper kinase (MELK) regulates multipotent neural progenitor proliferation. J Cell Biol 170:413-427

11. Nakano I, Masterman-Smith M, Saigusa K et al (2008) Maternal embryonic leucine zipper kinase is a key regulator of the proliferation of malignant brain tumors, including brain tumor stem cells. J Neurosci Res 86:48-60

12. Facchino S, Abdouh M, Chatoo W et al (2010) BMI1 confers radioresistance to normal and cancerous neural stem cells through recruitment of the DNA damage response machinery. J Neurosci 30:10096-10111

13. Kanamori M, Kawaguchi T, Nigro JM et al (2007) Contribution of Notch signaling activation to human glioblastoma multiforme. J Neurosurg 106:417-427

14. Stockhausen MT, Kristoffersen K, Poulsen HS (2010) The functional role of Notch signaling in human gliomas. Neurooncology 12:199-211

15. Marie SK, Okamoto OK, Uno M et al (2008) Maternal embryonic leucine zipper kinase transcript abundance correlates with malignancy grade in human astrocytomas. Int $\mathrm{J}$ Cancer 122:807-815

16. Dunn IF, Heese O, Black PM (2000) Growth factors in glioma angiogenesis: FGFs, PDGF, EGF, and TGFs. J Neuro-oncol 50:121-137

17. Mentlein R, Forstreuter F, Mehdorn HM et al (2004) Functional significance of vascular endothelial growth factor receptor expression on human glioma cells. J Neuro-oncol 67:9-18

18. Huang H, Held-Feindt J, Buhl R et al (2005) Expression of VEGF and its receptors in different brain tumors. Neurol Res 27:371-377

19. Furnari FB, Fenton T, Bachoo RM et al (2007) Malignant astrocytic glioma: genetics, biology, and paths to treatment. Genes Dev 21:2683-2710

20. Zhang M, Song T, Yang L et al (2008) Nestin and CD133: valuable stem cell-specific markers for determining clinical outcome of glioma patients. J Exp Clin Cancer Res 27:85

21. Xu P, Yu S, Jiang R et al (2009) Differential expression of Notch family members in astrocytomas and medulloblastomas. Pathol Oncol Res 15:703-710

22. Abdouh M, Facchino S, Chatoo W et al (2009) BMI1 sustains human glioblastoma multiforme stem cell renewal. J Neurosci 29:8884-8896

23. Phillips HS, Kharbanda S, Chen R et al (2006) Molecular subclasses of high-grade glioma predict prognosis, delineate a 
pattern of disease progression, and resemble stages in neurogenesis. Cancer Cell 9:157-173

24. Brennan C, Momota H, Hambardzumyan D et al (2009) Glioblastoma subclasses can be defined by activity among signal transduction pathways and associated genomic alterations. PLoS One 4:e7752
25. Verhaak RG, Hoadley KA, Purdom E et al (2010) Integrated genomic analysis identifies clinically relevant subtypes of glioblastoma characterized by abnormalities in PDGFRA, IDH1, EGFR, and NF1. Cancer Cell 17:98-110 